\title{
Toward an Electromagnetic Paradigm for Biology and Medicine
}

\author{
ABRAHAM R. LIBOFF, Ph.D.
}

\begin{abstract}
Work by Lund, Burr, Becker, and others leads to the inescapable conclusion that organisms tend to express quasisystemic electric changes when perturbed, and, conversely, will tend toward wellness either through enहेdogenous repair currents or the application of equivalent external currents. We show that an all-inclusive electromagnetic field representation for living systems is fully consistent with this extensive body of work. This 昰electrogenomic field may provide the basis for a new paradigm in biology and medicine that is radically difforent from the present emphasis on molecular biology and biochemistry. An electromagnetic field description also enables a more rational transformation from the genome than the present endpoint, universally stated in S్ țterms of the so-called visible characteristics. Furthermore, once the organism is described as an electromagOैnetic entity, this strongly suggests the reason for the efficacy of the various electromagnetic therapies, namely Eas the most direct means of restoring the body's impacted electromagnetic field to its normal state.
\end{abstract}

\section{IS MOLECULAR BIOLOGY THE FINAL WORD?}

gheld sway for more than 100 years. Some 50 years ago, Watstson and Crick extended the emphasis on biochemistry to inôclude DNA/RNA. The functioning of the body, its problems, चू.and repairs, are now completely formulated in terms of biomolecules and their interactions. Today the medical com@munity takes for granted that the best way to describe the living state is in terms of molecular biology, and that questions of illness and wellness must be ultimately answered in this context.

How does electromagnetic therapy fit into this picture? Is it merely one more convenient physical tool useful sometimes in leveraging physiologic adjustments? Or does it fit the phrase often associated with Feynman: is it a new way of looking at the biologic world? Is electromagnetic therapy indicative of a strikingly different paradigmatic shift?

The medical community is so fixed in its ways that it does not quite know what to do with electromagnetic therapy. This is despite the small but increasing acceptance of electromagnetic techniques by clinicians in recent years. An out- standing example was the use of pulsed magnetic fields (Bassett et al., 1974) followed by ion cyclotron resonance magnetic field combinations (Diebert et al., 1994) to treat bony nonunions. Another has been the use of rapid transcranial magnetic stimulation (rTMS; Barker et al., 1985) to treat depression. One problem for the clinician is that there is no rationale to fall back on to provide guidance in choosing among the variety of electromagnetic devices. With pharmaceuticals, for example, the physician can at least make an educated guess. But there is no underlying theory connecting electromagnetics to physiology. To add to the problem, we find clinicians lacking even a rudimentary understanding of electricity and magnetism.

In an attempt to find a reasonable way of classifying the variety of neuroelectromagnetic therapies presently being studied, we (Jenrow and Liboff, 2003; Liboff and Jenrow, 2002) concluded that based on the level of current densities produced, such therapies readily fall into three categories: disruptive, gross, and subtle. The self-descriptive term disruptive is illustrated by electroshock therapy or its newer magnetic equivalent, rTMS. Gross therapies are those that apply electromagnetic signals to mimic or recreate in-house physiologic signals that have gone awry, pacemakers being the prime example. The third category, subtle, is reserved

Department of Physics, Oakland University, Rochester, MI, and Center for Molecular Biology and Biotechnology Florida Atlantic University Boca Raton, FL. 
for signal strengths that are seemingly too small to be coupled to any known physiologic events, much less capable of disrupting anything in the nervous system. Physicists tell us that there are lower bounds to the effectiveness of electric or magnetic applications, bounds that are well characterized because of their profound understanding of the electrical signals generated by random thermal vibrations in molecules, including the molecules that are found in living systems. Nonetheless, reality in science is based on what you observe, not on what you are supposed to observe. Just as Galileo was alleged to have muttered that the earth moves around the sun despite the opinion of the Court of the Inquisition, many subtle electromagnetic effects are based in reality.

How then to explain the basic nature of these and other bioelectromagneticinteractions? In my opinion, much of the research directed at this question falls short of the mark. The existing medical paradigm is so pervasive that it seems natప̂ural to regard such EM interactions as convenient adjuncts "to the "normal" biochemical mechanisms by means of

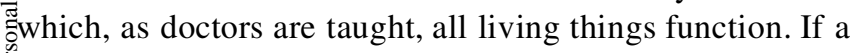
dxertain voltage with a certain waveshape can enhance serotonin levels or increase activation rates in calcium-binding Siproteins or increase the expression of heat shock protein, this becomes an end in itself. Rare indeed is the physiolotgist who inquires as to why this happens. The applied EM ợsignal results, in this view, in changes that physicians can comprehend, changes that can readily be tied to the accepted medical paradigm. It matters little how these signals do the trick. Electromagnetism merely serves to transform the thersapy into the familiar language of biochemistry. The medical o. community, the pharmaceutical industry, the funding agenicies, and the health care providers have all been weaned on inthis language, and, because they are uncomfortable with ج్lectromagnetism, they are content to see the results phrased in terms of hormones, cytokines, and membrane receptors. I believe there is a second way of interpreting such reos sults, one that leads to a totally different paradigm, one that is based on a language as rich and varied as the familiar biochemistry and molecular biology. Simply stated, it is possible to view the living system as an electromagnetic entity, with the response of the system to a given electric or magnetic signal as an outcome expected on the basis of physical law. The familiar hormonal and enzymatic effects still occur, but in this new approach these are merely associated changes in the system. Viewed in this light, I think that in the future biochemical responses are likely to be regarded as less fundamental than the corresponding changes in the electromagnetic state of the system.

\section{LIFE AS AN EXPRESSION OF THE ELECTROMAGNETIC FORCE}

Is there any evidence that such a description exists? To begin with, our understanding of nature tells us that there are likely no more than four types of forces in the universe, one of which is the gravitational and another the electromagnetic. I prefer to think of living things as direct and necessary consequences of the existence of electromagnetic force. Just as our planetary system and the structure of galaxies are necessary consequences of the gravitational force, the electromagnetic force is the underlying reason for life. Just as atoms are higher order collections of electrons and hadrons, and molecules are collections of atoms, and polymers are collections of molecules, so too, life is an interactive assembly of polymers. One merely has to consider the sequence of increasingly complex systems, each necessitated by the existence of the electromagnetic force, a sequence that directly leads to life: electrons, hadrons, atoms, molecules, polymers, living things.

\section{HINTS OF ELECTROMAGNETIC ORGANIZATION IN BIOSYSTEMS}

There are, however, more salient clues. Two separate types of widespread experimental evidence hint at some sort of electromagnetic organization in living things. First, there are studies indicating many remarkable intrinsic electrical features associated with biostructures, features that are, in some cases, directly tied to biological functions such as development, growth, and repair. The second type of clue is generated by the surprising fact that living things are sensitive to external electromagnetic fields, often at intensities so weak as to raise questions of credibility.

\section{ENDOGENOUS CURRENTS}

The idea that the presence of endogenouselectric and magnetic signals might be indicative of hitherto undiscovered levels of organization in living systems is not exactly a new concept. Since the time of Carlo Matteucci, a professor of physics in the early nineteenth century, it has been known that when the integrity of living tissue is perturbed, as in amputation or other injury, electric currents are generated in the vicinity of the problem area. These currents are referred to as currents of injury (Loeb and Beutner, 1912). After a provocative experiment on such currents in plants by Siniukhin (1957), Becker made a brilliant conceptual leap (Becker, 1974). After observing during salamander regeneration that after some time the injury current reverses polarity, he concluded that this reversal signals the dominance of healing over injury. This in turn implies the existence within living things of a complex electrically based process that first, signals that there is a problem, and second, provides another signal to repair things.

During the 1920s and 1930s, Lund (1947) showed that plants exhibit a remarkably well-defined endogenous electric dipole field. Pohl (1981) later reinforced this discovery by observing a similar dipole electric field in living cells in culture, lending credence to the concept that the organization of organisms may be electrically mediated. Clinical observations 
and speculations concerning the potential relation between sickness and endogenous electricity were made by Burr at Yale University. He reported finding links between various pathologies and the electric surface potentials of the impacted organs (Burr, 1972). These correlations were generalized (Burr and Northrup, 1935) into a sort of electric template for wellness (the L-field) based on the surface distribution of voltage. However, in general it can be said that, except for a few isolated individual researchers, primarily Becker and Selden (1985) and Athenstaedt (1969), the scientific community has all but ignored the work of Lund and Burr.

A separate series of experiments on hard tissue by Fukada and Yasuda (1957) established that bone has a well-characterized piezoelectric property, the unique characteristic that transforms mechanical stress to an electric potential difference. Later, Fukada and Yasuda (1964) showed that this piezoelectric property resulted from the interspersed collazgen component in bone, and it was subsequently suggested (Shamos and Lavine, 1967) that this decidedly physical

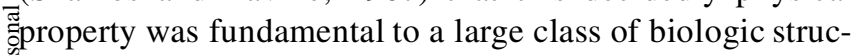
gัtures. Subsequent detailed measurements on the piezoelecîtric effect in intact long human bone (McElhaney, 1967) तुwere interpreted by Marino and Becker (1970) as showing that daily forces such as walking and mechanical support, wwhen transmitted to undifferentiated cells, determined ठิWhether these cells became osteoclasts or osteoblasts, bone icells that act to either destroy or grow bone, respectively. LLong before anyone suspected that bone contained functional electrical properties, it was known that bone continJually remodels itself to provide optimal mechanical support 气్ํfor its load bearing (Wolff's law). Thus, Marino and Becker (1970), using the electrical properties of bone, succeeded in Sexplaining an orthopedic principle that dated from the latIjter half of the nineteenth century. Their electromechanical control system is best illustrated in the osteopenia suffered चुby astronauts following lengthy periods of weightlessness. Another on-board electric regulatory mechanism has also been identified for developing organisms. Building on Lund's original work (1947), Athenstaedt (1969) found a clear tie-in between electric polarization in the skeleton and human development. This finding was reinforced by Friedenberg et al. (1973). In studies on developing bone, they reported that a well-characterized electric potential is specifically associated with the growth plate in long bone.

These and other observations clearly indicate that electricity, either endogenously available or occurring as the result of internal transduction processes, is used to physiologic advantage in living systems, in guiding growth, repair, and regeneration.

\section{THE RESPONSE TO APPLIED ELECTROMAGNETIC FIELDS}

Nearly coincident with these reports of endogenous electrical characteristics in biologic systems, others discovered that organisms exhibit the obverse: they also respond biologically to applied electromagnetic fields. One persistent idea followed the original concept by Lund (1947) that small currents may aid or mimic or oppose naturally occurring endogenous signals arising either as "currents of injury" or in connection with growth and development. If the growth in living systems is engineered in situ by endogenous currents, why not apply external signals to do the same? Thus currents were used (Becker, 1972; Smith, 1967) to assist in forcing limb regeneration in rats. Another approach similar to but not quite the same as in the current of injury concept was due to Nordenstrom (1983) who hypothesized the existence of intrinsic electric pathways in the body, the most prominent being the low-resistance vascular network. Based on his Biologically Closed Electric Circuits (BCES) hypothesis, Nordenstrom suggested that endogenous currents appear as a consequence of pathological disorders, and thus it should be possible to treat such disorders with properly applied electrical signals. Nordenstrom's concepts were extended (Chou, 1997; Xin et al., 1997) to electrotherapeutic techniques to treat (with some success) a variety of malignant tumors.

A great deal of work originally centered on the likelihood that biologic systems have probably evolved to use the earth's magnetic field to their own advantage. As one excellent example in a wide literature base (e.g., Presman, 1970) on this subject, Brown (1962) determined that planaria and other organisms are sensitive to changes in the geomagnetic field.

Furthermore, if growth and remodeling in bone are the result of electricity, why not use electricity to enhance bone growth and repair? The original discovery by Yasuda (1953) that small direct current (DC) electric currents applied to living bone results in callus formation, even in the absence of a fracture, led to studies on the potential use of such currents in treating bony nonunions (Lavine et al., 1972). Bassett et al. (1974) demonstrated that these currents were equally effective when they were induced by pulsed magnetic fields generated by coils situated close to the defect. Similar levels of efficacy resulted when ion cyclotron resonance (ICR) combinations of alternating current (AC) and DC magnetic field (Diebert et al., 1994) were used. Presently, Food and Drug Administration (FDA)-approved devices using both techniques are prescribed to speed recalcitrant nonunions and spinal fusion.

Another group of electromagnetic techniques has been developed for neurotherapies. There are presently (Jenrow and Liboff, 2003) approximately 10-12 different electromagnetic devices for treating neurologic and behavioral problems, with a wide range of applied currents, frequencies, and waveshapes.

In the 1980s, two things tended to spur greater interest in the response of biologic systems to magnetic fields. First, there was the possibility that $50 / 60 \mathrm{~Hz}$ electrical transmission and distribution presented a hazard, particularly with respect to the induction of childhood leukemia (Wertheimer 
and Leeper, 1979). Second, the fact that pulsed magnetic fields were found to play a role in repairing bone led to studying the effects of such fields in a wide variety of other systems. For example, Cossarizza et al. (1989) observed that lymphocytes from aged humans, when exposed to pulsed magnetic fields, tend to recover much of the immune loss that ordinarily occurs with aging. Using both pulsed magnetic fields (Goodman et al., 1983) and sinusoidal magnetic fields (Goodman and Blank, 2002) Goodman and coworkers have repeatedly reported electromagnetic-induced genomic changes, particularly the expression of heat shock stress proteins.

An important chapter in studying the biologic response to applied EM fields was opened by Suzanne Bawin, working in Ross Adey's laboratory (Bawin and Adey, 1976). For the first time a response was obtained that appeared to carry a more physically meaningful message, in that maximum biologic responses were observed at certain frequencies. This כैwas suggestive of resonances observed in many nonliving 伺ystems. This response was subsequently identified (Liboff एँand McLeod, 1988) as resulting from the Lorentz force, an interaction specifically requiring the movement of charged î.particles (e.g., ions) in a magnetic field. This can give rise (Liboff, 1985) to the ICR response. Since that time, this type Eof resonance has been observed not only in bone, but also ơ.in rat behavior, diatom motility, calcium uptake in cell cultiture, neurite outgrowth, in plant growth, and in other or.g ganisms (Liboff, 2003).

\section{AN ELECTROMAGNETIC FIELD FOR THE ORGANISM}

Thus, the past 50 years have revealed both that there is a remarkable endogenous electric character to organisms, and, galso, that there are equally remarkable effects in biologic ơ systems when they are exposed to electromagnetic fields. DNevertheless, as imaginative and innovative as the various experiments, starting with Lund, may have been, they have failed to come to grips with the larger question: What is the nature and meaning of the electric character that seems to permeate living things?

The author wrote in 1994 (Liboff, 1994):

... does the ability of weak ELF signals to perturb living systems indicate that these signals are interacting with an intrinsic electromagnetic characteristic of these systems? . . . do living systems contain a hidden electromagnetic variable?

One way to explain the experimental results is to invoke a special electromagnetic field for all organisms, a field that is not epiphenomenal but serves a specific biologic function.

It happens that it is possible to connect living things in an unequivocal manner to electromagnetism. This connec- tion is based on physical law. It is not a matter of arbitrary choice but rather a matter of definition. Electromagnetic fields are always precisely defined in terms of what are referred to as source densities. An electromagnetic field only exists when the system contains source distributions of charge density and current density. Every living thing carries these two sources, which ensures that every living thing, by definition, also carries an associated electromagnetic field (Liboff, 1994). But there is more. Unlike nonliving entities, charge and current sources in living things are distinctive. They are arranged in ways through natural selection that allow the organism to survive. With death, these distributions of charge and current, or equivalently, the associated electromagnetic field for the system, are no longer viable. In brief, we are suggesting that the life process itself is an expression of the electromagnetic field.

One interesting consequence of this is that it is possible, in principle, to formulate a specific electromagnetic field that in essence represents a specific organism. Rather than concentrate on the electrical characteristics of any one component of the body, we associate the entire organism with the field. Elsewhere (Liboff, 1994, 1996), it has been shown how such a field can be mathematically described in terms of a vector $\Pi$ that is a function of space and both ontogenic and phylogenetic times. This field grows with us as we develop and age, and is itself the product of evolution, relating each of us to our species and all that came before. The field reflects the changes in the developing embryo, and in the way we develop towards maturity after birth. It reflects the traumatic changes associated with wounds. Also, following Athenstaedt (1969) and Becker (1974), not only do these field changes reflect the changes in source density, but the changed field acts as the template for restoring the system to its normal state. Most relevant to electromagnetic therapy, the conclusion that living things are expressions of the electromagnetic field explains electromagnetic medicine in a new way: we can change the intrinsic electromagnetic field when external electromagnetic fields are applied. Once we admit to the possibility that the gestalt of the body's physiologic state-homeostasis, metabolic turnover, respiration, enzymatic rates--is no more than an intertwined system that can be represented by a single electromagnetic field vector, then we are also admitting to the fact that this intrinsic field and therefore its corresponding physiologic state will be changed by imposing a new applied field. Needless to point out, the change can either be beneficial in the form of a therapeutic signal to repair that which has gone awry, or it can be harmful, as an unwanted deviation from the normal resting state. Therefore, the formulation of this field also subsumes the problem of electromagnetic pollution, and likely, the question of electrosensitivity as well. There should be no question among those making use of electric or magnetic therapies that humans can react negatively to the imposition of electromagnetic fields that act to distort the body's normal electromagnetic field. 


\section{REPLACING THE VISIBLE CHARACTERISTICS}

It must be emphasized that this field is not merely associated with the living system, but may itself be the ultimate biologic representation of the system. For centuries, well before Darwin, biologists have delineated each organism by means of its characteristics, more properly referred to as its visible characteristics-its shape and size, the location of the eyes, the color of flowers, the shape of beaks, the number of fins, the inner components of the body, and all the other myriad aspects that constitute a precise means of distinguishing an organism from other species and from other members of the same species. The discovery of DNA/RNA was used to explain these characteristics in the sense that there is (almost) a one-to-one correspondence between a specific characteristic and an element or elements in the genome. In mathEematical terms, this relation between the genome and the ơ๊visible characteristics can be referred to as a transformation-in the simplest definition, a transformation allows ione to express the same thing in two ways when viewed Sin two different coordinate systems. In principle, for each fvisible characteristic that one can think of, there is a corwresponding element of the genome that carries specific oinformation relevant to this characteristic. However, this हैway of describing every living thing in terms of its visioble characteristics is a flawed concept. Nowhere else in sscience do we allow descriptions of what we are measuring to be so unabashedly subjective. Equally importhant, how does one get a mathematical handle on long Raundry lists of disparate things like skin, eyes, and size? PPhysics, probably because it was forced to deal with enfities that could not be seen, developed mathematical thechniques (some would say borrowed mathematical techInniques), and the results have been spectacular, because Fुonce there is a good mathematical handle at your disoposal, then one can use this to hunt for the special relations that may exist for the system. Some have stated this problem by arguing that biology will only be understood when we develop or discover the missing "Newton's laws of biology," the mathematically distinct rules from which everything that can be known about biologic systems can be discerned.

Thus, when we postulate the existence of an electromagnetic field that is distinctly representative for each and every organism, we are also arguing first, that this representation may be more mathematically accessible than the present system using visible characteristics, and second, that a more fertile basis will result from the transformation that relates the genome to this field, as opposed to the existing, rather sterile transformation relating genome to characteristics. Because we are relating the characteristic electromagnetic field of an individual to the individual's genome, we will call this field the electrogenomic field.

\section{SUGGESTED POSTULATES}

In reviewing the work exploring the relation between electromagnetism and biology, we find that this work strongly suggests an overarching explanation that is purely fielddriven. On the one hand, when the system is away from equilibrium, either while still developing or as the result of injury, the system expresses electric current in a functional way, as part of the growth or repair mechanism. What is especially provocative is that this expression is in terms of quasi-systemic currents, emanating from large areas of the system, connecting enormous numbers of cells, and quite clearly representing a process that is more than the sum of its parts.

On the other hand, when one applies external voltages or otherwise applies currents to large areas of tissue, also covering a multitude of cells, specific physiologic responses occur. These two aspects, the intrinsic functional currents and the response to equivalent external currents, taken together, are quite remarkable, especially considering that in both cases the component cells are acting in concert.

The only explanation for all of this is that the living system enjoys a characteristic electric field that is somehow intrinsically interwoven into the fabric of the system, a field that will generate various local currents in growth, in stasis, and in repair modes. Although the field we have suggested is electric and quasistatic, it is reasonable to extend the notion to the most general case (i.e., a field that is time varying and electromagnetic).

We therefore present the following two postulates:

1. Every living organism is completely described by an electromagnetic field vector $\Pi_{0}$ that is specifically determined by a transformation from the genome.

2. All pathologies, abnormalities and traumas are manifested by deviations from the normal field $\Pi_{0}$, and, within limits, these deviations are compensated for by the homeostatic tendency of the system to return to $\Pi_{\mathrm{o}}$.

\section{DISCUSSION}

The concept that some sort of lawful generalization governs living things is certainly not a new idea (Goodwin, 1989; Waddington, 1972). Many such attempts however, remain deeply rooted in biology, searching for rules implicit in the way that biologic systems function, for example, their self-replicating properties (Turing, 1952). Even the morphogenetic field proposed by Sheldrake (1988) is completely biologic in flavor, stressing the visible characteristics of the organism. Remarkably, none of these approaches to the biofield are necessarily connected to the electromagnetic field. This, despite the strides of those such as Lund (1947), Burr (1972), Becker (1974), and Athenstaedt (1969) who collectively demonstrated how deeply electricity is involved in the organization and functioning of living things. 
More recently, the heightened interest in energy medicine has to some extent focused on the possibility that the biofield may have an electromagnetic basis (Rubik, 2002). This argument is based on the fact that the effects observed in both energy medicine and bioelectromagnetism are unusually subtle, an interesting observation, but by no means proof that there is a connecting link. Although there is no evidence to date that shows this connection, the postulates that we propose provide a good working premise with which to further explore energy medicine.

There are a number of additional interesting implications embodied in these postulates. Electromagnetic fields enjoy the common property that the field is never limited to the boundary of the system of sources (in living things, the skin). There is ample proof that electromagnetic fields are generated in humans and that these extend beyond the skin. The military uses night-vision devices for remote viewing perపsonnel. Medical diagnostic procedures make use of infrared thermography for subcutaneous examination. Weak miప્ّ crowave signals are radiated by living things. However these oncases, involving infrared and microwave detection, simply ثैreflect the fact that any heated object will radiate. But aniIimals also emit another type of electromagnetic signal. SuFेperconducting quantum interference detection (SQUID) stechniques routinely detect magnetic signals from the brain, țthe heart, and other endogenous current sources. Each of these signals is magnetically coherent, reflecting the fact that the currents in each source are in phase, with changes occurring simultaneously.

Because of this we find the potential for biocommunicaड़ํion, more specifically intersystem electromagnetic bioicommunication. The electromagnetic characteristics of livNing things may therefore allow for direct communication क् recent evolutionary development of speech. The primary offactor required for this would be signal intensity, not in the ơ form of broadband thermal radiation, but more frequency ospecific. The oscillator serving as the basis for the underlying time-varying electromagnetic field must be coherent in order for information to be transmitted. Remarkably, there are compartments in our brains that reflect just this-specific frequencies. Among such sources are those producing $\gamma$-oscillations (Singer, 1993) reflecting millions, perhaps billions, of neuronal elements all oscillating in phase.

In recent years, some (Behe, 1998) have questioned the viability of Darwinian evolution, claiming that certain biologic structures are so complex (e.g., the eye) that they could not have been formed over the several billion years everyone agrees transpired since life began. However, this argument, often termed irreducible complexity, is deeply rooted in the molecular biology paradigm. Behe's argument becomes far less of an issue if organizational imperatives exist other than those found in traditional biology. In particular, the presence of the electrogenomic field mitigates this argument. Biologic structures that are biochemically com- plex need not be electromagnetically complex. On the contrary, there is a far greater simplicity in using an electromagnetic field to describe living things compared to the widely disparate distribution of enzymes and other biochemical factors that are normally used. This reduction of biologic complexity is a direct consequence of the mathematical abstract that we call field, in much the same way as Faraday and Maxwell used the field concept to simplify electricity and magnetism.

One of the most persistent opinions of those advocating alternative medicine is that the ailing body should be treated holistically. One finds this in approaches that, among many others, attempts to balance yang and yin, involve meditation or the practice of yoga, and especially in Western culture, promote the idea that certain regimens, like daily exercise or eating yoghurt, are good for you. With the electrogenomic field, one finds for the first time a potential basis for holistic biology and medicine that is entirely framed in terms of existing physical law.

Finally, we note the direct manner in which electromagnetic therapy can be folded into the postulates above. Instead of providing a tool that affects the variables that are part of the existing medical paradigm, electromagnetic therapy is revealed as the most direct means of restoring the fundamental electromagnetic parameters of the body. It is also clear that electromagnetic therapies are not to be used haphazardly but require some thought in their implementation.

\section{REFERENCES}

AthenstaedtH. Permanent electric polarization and pyroelectric behavior of the vertebrate skeleton III. The axial skeleton of man. Z Zellforsch 1969;93:484-504.

Barker AT, Jalinous R, Freeston JL. Non-invasive magnetic stimulation of human motor cortex. Lancet 1985;1:1106-1107.

Bassett CAL, Pawluk RJ, Pilla AA. Acceleration of fracture repair by electromagnetic fields: A surgically noninvasive method. Ann NY Acad Sci 1974;238:242-262.

Bawin WM, Adey WR. Sensitivity of calcium binding in cerebral tissue to weak electric fields oscillating at low frequency. Proc Natl Acad Sci USA 1976;73:1999-2003.

Becker RO. Stimulation of partial limb regeneration in rats. Nature 1972;235:109-111.

Becker RO. The significance of bioelectric potentials. Bioelectrochem Bioenergetics 1974;1:187-199.

Becker RO, Selden G. The Body Electric. New York: Morrow, 1985.

Behe MJ. Darwin's Black Box. Touchstone, New York, 1998.

Brown F. Response of the planarian Dugesia and the protozoan Paramecium to very weak horizontal magnetic fields. Biol Bull 1962;123:264.

Burr HS, Northrup FSC. The electro-dynamic theory of life. Q Rev Biol 1935;10:322-333.

Burr HS. The Fields of Life. New York: Ballantine Books, 1972. Chou CK. Electrochemical treatment of tumor. Bioelectromagnetics 1997;18:1. 
Cossarizza A, Monti D, Bersani F, Paganelli R, Montagnani G, Cadossi R, Cantini M, Franceschi C. Extremely low frequency pulsed electromagnetic field increase interleukin-2 (IL-2) utilization and IL-2 receptor expression in mitogen-stimulated human lymphocytes from old subjects. FEBS Lett 1989;248:141144.

Diebert MC, McLeod BR, Smith SD, Liboff AR. Ion resonance electromagnetic field stimulation of fracture healing in rabbits with a fibular ostectomy. J Orthop Res 1994;12:878-885.

Freidenberg ZB, Harlow MC, Heppenstall RB, Brighton CT. The cellular origin of bioelectric potentials in bone. Calcif Tissue Res 1973;13:53-62.

Fukada E, Yasuda I. On the piezoelectric effect in bone. J Phy Soc Japan 1957;12:1158-1162.

Fukada E, Yasuda I. Piezoelectric effects in collagen. Jpn J Appl Phys 1964;3:117-121.

Goodman R, Bassett CAL, Henderson AS. Pulsing electromagnetic fields induce cellular transcription. Science 1983;220:1283-1285.

Goodman R, Blank M. Insights into electromagnetic interaction

Dechanisms. J Cell Physiol 2002;192:16-22.

Goodwin BC. Evolution and the generative order. In: Goodwin B,

Saunders P, eds. Theoretical Biology: Epigenetic and Evolu-

tionary Order from Complex Systems. Baltimore: Johns Hop-

芒 kins, 1989.

กิJenrow KA, Liboff AR. Electromagnetic techniques in neural therapy. In: Rosch PJ, Markov M, eds. Bioelectromagnetic Medicine. New York: Dekker, 2003.

ELavine LS, Lustrin I, Shamos MH, Rinaldi R, Liboff AR. Electric enhancement of bone healing. Science 1972;175:1118-1121.

Loeb J, Beutner R. The source of the current of injury. Science : 1912;35:970-971.

Liboff AR. Geomagnetic cyclotron resonance in living cells. J Biol Physics 1985;13:99-102.

Liboff AR. The electromagnetic field as a biological variable. In: Fे Frey AH (ed.) On the Nature of Electromagnetic Field Interacกิ tions. Austin: R.G. Landis, 1994.

तेLiboff AR. Evolution and the change in electromagnetic state. in Electro- and Magnetobiology 1996;15:245-252.

DLLiboff AR. The charge-to-mass ICR signature in weak ELF bioelectromagnetic effects. In: Lin J, ed. Advances Electromagnetic Fields in Living Systems, Vol. 4. New York: Kluwer/Plenum, 2003.

Liboff AR, Jenrow KA. Physical mechanisms in neuroelectromagnetic therapies. Neurorehabilitation 2002;17:9-22.

Liboff AR, McLeod BR. Kinetics of channelized membrane ions in magnetic fields. Bioelectromagnetics 1988;9:39-51.

Lund EJ. Bioelectric Fields and Growth. Austin: The University of Texas Press, Austin, 1947.
Marino AA, Becker RO. Piezoelectric effect and growth control in bone. Nature 1970;228:473-474.

McElhaney JH. The charge distribution on the human femur due to load. J Bone Joint Surg 1967;49:1561-1571.

Nordenstron BEW. Biologically Closed Electric Circuits. Stockholm: Nordic Medical Publications, 1983.

Pohl HA, Braden T, Robinson S, Picardi J, Pohl DG. Life cycle alterations of the micro-dielectrophoretic effects of cells. J Biol Phys 1981;9:133-152.

Presman AS. Electromagnetic Fields and Life. New York: Plenum, 1970.

Rubik B. The biofield hypothesis: Its biophysical basis and role in medicine. J Altern Complement Med 2002;8:703-717.

Shamos MH, Lavine LS. Piezoelectricity as a fundamental property of living tissues. Nature 1967;213:267-269.

Sheldrake R. The Presence of the Past. New York: Times Books, 1988.

Singer W. Synchronization of cortical activity and its putative role in information processing and learning. Annu Rev Physiol 1993; 55:349-374.

Siniukhin AM. Nature of the variation of the bioelectric potentials in the regeneration process of plants [in Russian]. Biofizika 1957;2:52.

Smith SD. Induction of partial limb regeneration in Rana pipiens by galvanic stimulation. Anat Rec 1967;158:89-98.

Turing AM. The chemical basis of morphogenesis. Phil Trans Royal Soc 1952;B237:37-72.

Waddington $\mathrm{CH}$. Essays In: Waddington $\mathrm{CH}$, ed. Towards a Theoretical Biology, Vol. 4. Edinburgh: Edinburgh University Press, 1972.

Wertheimer N, Leeper E. Electrical wiring configurations and childhood cancer. Am J Epidemiol 1979;109:273-284.

Xin Y-L, Xue F-Z, Ge B-S, Zhao F-R, Shi B, Zhang W. Electrochemical treatment of lung cancer. Bioelectromagnetics 1997; 18:8-13.

Yasuda I. Fundamental aspects of fracture treatment. J Kyoto Med Soc 1953;4:395; translated from the original Japanese and reprinted in Clin Orthop 1977;124:5-8.

Address reprint requests to: Abraham R. Liboff, Ph.D.

Department of Physics Oakland University 272 Hannah Hall Rochester, MI 48309

E-mail: liboff@oakland.edu 